\title{
Synthesis of trisaccharides incorporating the $\alpha$-Gal antigen functionalized for neoglycoconjugate preparation
}

\author{
Patrice Plaza-Alexander and Todd L. Lowary* \\ Alberta Glycomics Centre and Department of Chemistry, The University of Alberta, Gunning- \\ Lemieux Chemistry Centre, 11227 Saskatchewan Drive, Edmonton, AB T6G 2G2, Canada \\ E-mail: tlowary@ualberta.ca
}

\section{Dedicated to Professor Richard R. Schmidt on the occasion of his $78^{\text {th }}$ anniversary}

\begin{abstract}
The synthesis of trisaccharides 1 and 2 , which contain the $\alpha$-D-galactopyranosyl- $(1 \rightarrow 3)-\beta$-Dgalactopyranosyl ( $\alpha$-Gal) motif, is described. A key step in the synthesis of the trisaccharides was the glycosylation of a monosaccharide acceptor with a disaccharide trichloroacetimidate donor. Subsequent modification of the products of this [2+1] glycosylation afforded $\mathbf{1}$ and $\mathbf{2}$, which contain an activated ester moiety suitable for reaction with, for example, proteins or amine-coated surfaces.
\end{abstract}

Keywords: $\alpha$-Gal antigen, imidate, neoglycoconjugate, activated ester, immune tolerance

\section{Introduction}

The $\alpha$-galactopyranosyl-(1 $\rightarrow 3)-\beta$-D-galactopyranosyl disaccharide motif, also known as the $\alpha$ Gal antigen, is found commonly on the surface of mammalian tissues with the exception of old world monkeys and humans. ${ }^{1}$ This antigen has long been identified as a contraindication for xenotransplantation. ${ }^{2}$ Although the problems associated with xenotransplantation have been largely overcome by the advent of $\alpha$-Gal knockout pigs, ${ }^{3,4}$ the role of $\alpha$-Gal epitopes in transplantation $^{5-7}$ and in several disease conditions ${ }^{8-11}$ is still under investigation. There is also growing interest in the use of bioconjugates bearing the $\alpha$-Gal epitope in cancer therapies ${ }^{12-15}$ and vaccine development ${ }^{16}$ to enhance immune responses. Access to synthetic $\alpha$-Gal epitopes is required to facilitate most of this research.

$\alpha-(1 \rightarrow 3)$-Galactosyltransferase knockout (GTKO) mice have been routinely used to study the immune response to carbohydrate antigens. ${ }^{17}$ Analysis of $\alpha-\mathrm{Gal}$ response in GTKO mice is considered a good approximation for the study of accommodation and immune tolerance induction in transplantation of ABO-incompatible organs in humans, ${ }^{18}$ as the $\alpha$-Gal epitope is 
found in the A and B blood groups. ${ }^{19}$ As part of a larger investigation on the preparation of glycosylated nanomaterials ${ }^{20}$ for use in generating immune tolerance against carbohydrate antigens in neonates, we had the need for $\alpha$-Gal-containing oligosaccharides functionalized with an activated flexible linker, which would be used for the attachment to various surfaces. We describe here the synthesis of two such targets, trisaccharides 1 and $\mathbf{2}$ (Figure 1).

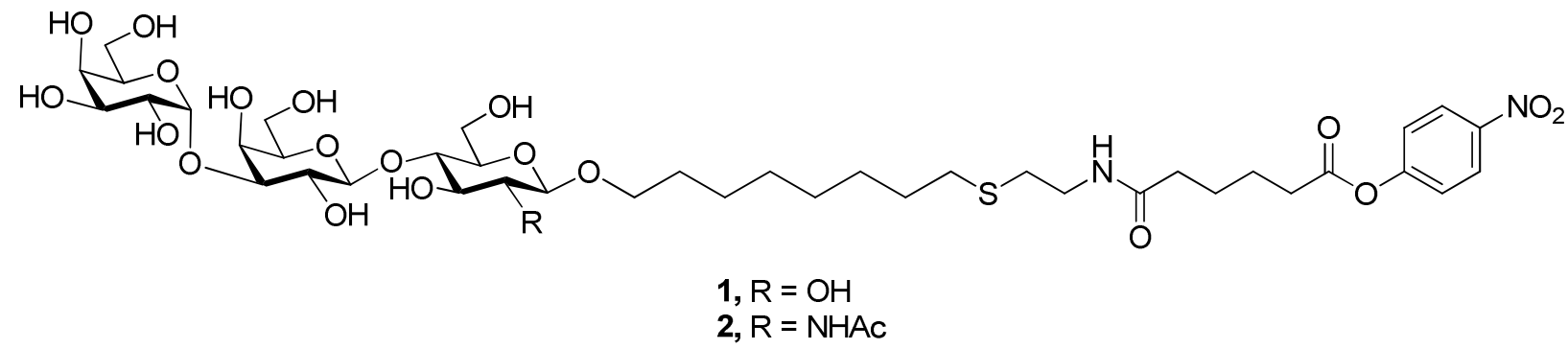

Figure 1. Structure of targets 1 and 2.

\section{Results and Discussion}

Production of $\alpha$-Gal epitopes for biological studies is often achieved by chemoenzymatic means. $^{21-23}$ Access to these compounds via this approach involves the use of recombinant $\alpha$ $(1 \rightarrow 3)$-galactosyltransferase enzymes and chemically modified substrates. Although this method is efficient and provides sufficient material for biological assays, access to larger quantities of material still favours chemical synthesis.

Several groups have reported the chemical synthesis of oligosaccharides incorporating the $\alpha$-Gal epitope. ${ }^{24-28}$ For example, Wang and coworkers achieved the synthesis of an $\alpha$-Gal pentasaccharide via a one-pot strategy using various thioglycoside donors. ${ }^{24,25} \mathrm{Schmidt}$ and coworkers described the synthesis of a related pentasaccharide using a convergent approach and trichloroacetimidate donors. ${ }^{27}$ In another investigation, Litjens et al. reported the one-pot synthesis of an $\alpha$-Gal trisaccharide using thioglycoside donors. ${ }^{26}$ Most previously reported synthetic routes to the trisaccharides such as $\mathbf{1}$ and $\mathbf{2}$ involve the selective protection and glycosylation of a lactose or lactosamine acceptor. We decided instead to prepare an $\alpha$-Gal $p$ $(1 \rightarrow 3)$-Gal $p$ disaccharide donor as a precursor and then react it with a monosaccharide acceptor. This would allow us to change the acceptor thus allowing for a straightforward convergent approach to the preparation of $\alpha$-Gal-containing tetra- and pentasaccharides at a later stage.

The preparation of $\mathbf{1}$ and $\mathbf{2}$ was accomplished using trichloacetimidate donors developed originally by Schmidt and co-workers. ${ }^{29}$ First, the $\alpha$-Gal $p$ - $(1 \rightarrow 3)$-Gal $p$ linkage was achieved in good yield and stereoselectivity by reaction of tricholoroacetimidate $\mathbf{3}$ and thioglycoside alcohol 4 using diethyl ether as a solvent (Scheme 1). ${ }^{30}$ To perform this reaction, thioglycoside $\mathbf{4}$, which was sparingly soluble in diethyl ether, was dissolved in a binary mixture of diethyl ether and dichloromethane (10:1). Reverse dropwise addition of the trichloroacetimidate donor $\mathbf{3}$ to the 
solution of 4 at $-10{ }^{\circ} \mathrm{C}$ in the presence of TMSOTf as an activator gave exclusively the desired $\alpha$-linked product, $\mathbf{5}$, in $76 \%$ yield. The $\alpha$-stereochemistry of the newly formed linkage in $\mathbf{5}$ was readily apparent from its ${ }^{1} \mathrm{H}$ NMR spectrum; the value of ${ }^{3} J_{1^{\prime}, 2^{2}}$, was $3.0 \mathrm{~Hz}$. This reaction was easily conducted in gram scale quantities with no change in stereoselectivity, thus providing large amounts of material for further synthesis.

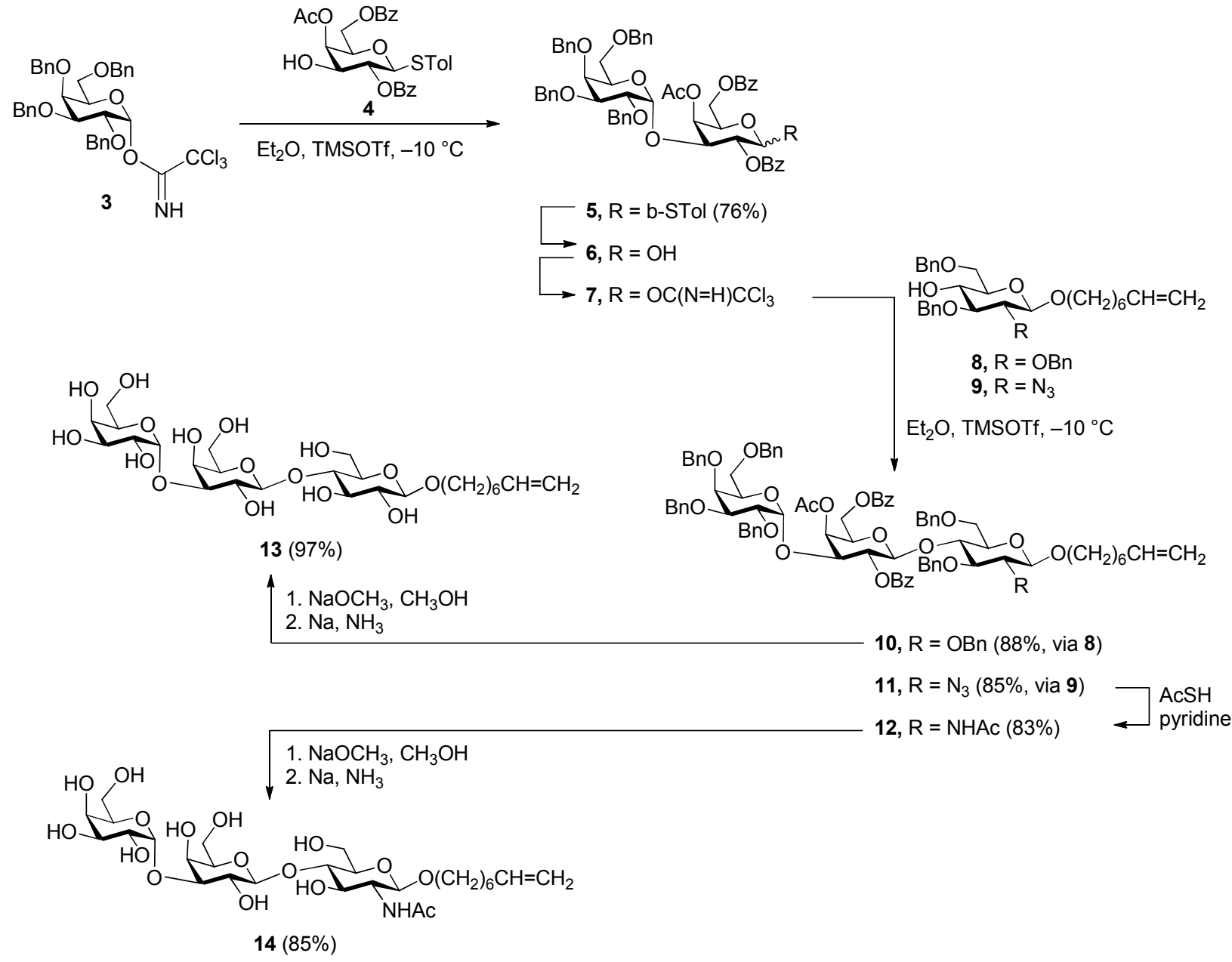

Scheme 1. Preparation of trisaccharides 13 and 14.

Direct coupling of the resulting thioglycoside $\mathbf{5}$ with the acceptors $\mathbf{8}$ and $\mathbf{9}^{31}$ could not be achieved, even in neutral conditions. We attribute this to the electrophilic by-products produced during the reaction, reacting with the alkene moiety in the acceptor. Thus, thioglycoside $\mathbf{5}$ was first transformed into the corresponding hemiacetal 6 in $84 \%$ yield, by the action of $N$ bromosuccinimide in acetone, and subsequently converted to trichloroacetimidate 7 by treatment with trichloroacetonitrile in DBU.

Coupling of trichloracetimidate 7 to either 8 or 9 proceeded uneventfully to give the corresponding trisaccharides $\mathbf{1 0}$ and $\mathbf{1 1}$ in 87 and 83\% yield, respectively. The $\beta$-stereochemistry 
of the newly formed bond in both molecules was confirmed by the ${ }^{3} J_{\mathrm{H} 1, \mathrm{H} 2}$ in $\mathbf{1 0}$ and $\mathbf{1 1}$, which were $8.1 \mathrm{~Hz}$ and $8.0 \mathrm{~Hz}$, respectively. The azido acceptor was chosen as an aminosugar precursor, primarily for ease of conversion to the desired $N$-acetyl group. This transformation could be achieved by reaction of $\mathbf{1 1}$ with thioacetic acid in pyridine, ${ }^{32}$ providing the expected acetamide derivative in $83 \%$ yield. Simultaneous deprotection of the benzoyl and benzyl protecting groups in $\mathbf{1 0}$ and $\mathbf{1 2}$ was carried out using dissolving metal reduction. This was achieved by reaction of each compound with sodium metal in $\mathrm{THF} / \mathrm{CH}_{3} \mathrm{OH}$, conditions that allowed the removal of the benzyl groups while leaving the alkene functionality intact. In addition, the benzoate esters were cleaved under the basic conditions of the reaction.

Having in place a robust route for the preparation of 13 and 14, we investigated their conversion into 1 and 2 . The octenyl linker of $\mathbf{1 3}$ and $\mathbf{1 4}$ was further functionalized by first introducing a cysteamine residue via a UV-promoted thiol-ene reaction ${ }^{33}$ to give the corresponding amines 15 and $\mathbf{1 6}$ as their hydrochloride salts in 90-97\% yield (Scheme 2). Further elaboration of the linker using di-p-nitrophenyl adipate ${ }^{34}$ in dimethylacetamide gave the highly reactive $p$-nitrophenol (PNP) esters 1 and $\mathbf{2}$. The final products were lyophilized and could be stored at $-20{ }^{\circ} \mathrm{C}$ without significant degradation over time.

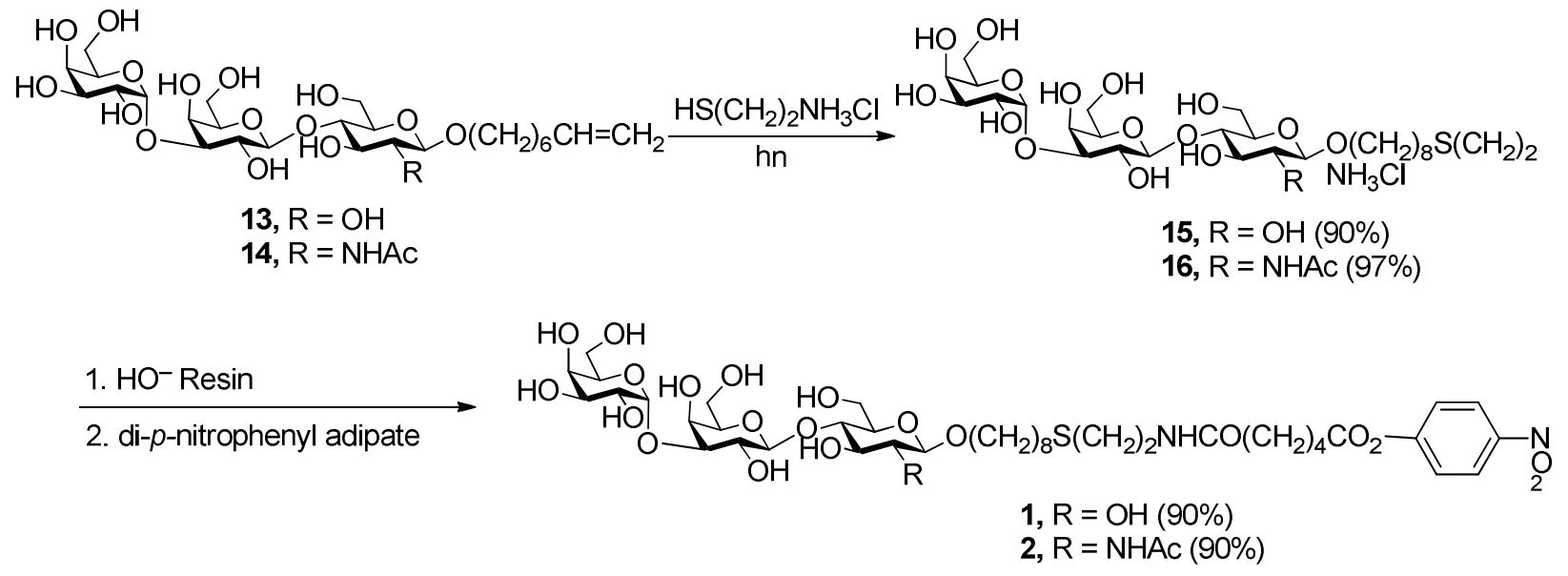

Scheme 2. Preparation of activated PNP ester derivatives 1 and 2.

\section{Conclusions}

In summary, we report an efficient route to $\alpha$-Gal trisaccharides $\mathbf{1}$ and $\mathbf{2}$ via a route in which a key transformation is the reaction of a disaccharide trichloroacetimidate donor with a monosaccharide acceptor. This route should be applicable to more complex glycans containing the $\alpha$-Gal antigen. The use of $\mathbf{1}$ and $\mathbf{2}$ in the preparation of nanoparticles containing this motif is underway and will be reported in the future. 


\section{Experimental Section}

General. Reagents and solvents were purchased from commercial sources and were used without further purification, unless otherwise stated. Dry solvents were purified by successive passage through columns of alumina and copper under nitrogen. Reactions were conducted in an inert atmosphere of argon gas, unless otherwise stated. Thin layer chromatography (TLC) was performed on E. Merck Silica Gel 60 F254 aluminum-backed plates that were stained by heating $\left(>200{ }^{\circ} \mathrm{C}\right.$ ) with either $p$-anisaldehyde in $5 \%$ sulfuric acid in EtOH or $10 \%$ ammonium molybdate in $10 \%$ sulfuric acid. Unless otherwise indicated, all column chromatography was performed on Silica Gel $60(40-60 \mu \mathrm{M}) . \mathrm{C}_{18}$ silica gel $(35-70 \mu \mathrm{M})$ was manufactured by Toronto Research Chemicals. Optical rotations were measured at $589 \mathrm{~nm}$, at $22 \pm 2{ }^{\circ} \mathrm{C}$ and are in units of $\mathrm{deg} \cdot \mathrm{dm}^{-1} \cdot \mathrm{cm}^{3} \cdot \mathrm{g}^{-1}$, in all cases the concentrations are in units $\mathrm{g} / 100 \mathrm{~mL} .{ }^{1} \mathrm{H}$ NMR spectra were recorded at $500 \mathrm{MHz}$, chemical shifts were referenced to the peak for TMS $\left(0.0 \mathrm{ppm}, \mathrm{CDCl}_{3}\right)$ or $\mathrm{CD}_{3} \mathrm{OD}$ (3.30 ppm, $\mathrm{CD}_{3} \mathrm{OD}$ ). ${ }^{13} \mathrm{C}$ NMR (APT) spectra were recorded at 125 or $100 \mathrm{MHz}$, and ${ }^{13} \mathrm{C}$ chemical shifts were referenced internal $\mathrm{CDCl}_{3}\left(77.1 \mathrm{ppm}, \mathrm{CDCl}_{3}\right)$ or $\mathrm{CD}_{3} \mathrm{OD}\left(49.0, \mathrm{CD}_{3} \mathrm{OD}\right)$. High resolution mass spectra were obtained using electrospray ionization in the appropriate solvents.

$p$-Tolyl 2,3,4,6-tetra- $O$-benzyl- $\alpha$-D-galactopyranosyl-( $\rightarrow 3$ )-4- $O$-acetyl-2,6-di- $O$-benzoyl-1thio- $\beta$-D-galactopyranoside (5). Acceptor thioglycoside 4 (1.5 g, $2.80 \mathrm{mmol}$ ) was dissolved in $\mathrm{Et}_{2} \mathrm{O}-\mathrm{CH}_{2} \mathrm{Cl}_{2}(10: 1 ; 55 \mathrm{~mL})$ containing $4 \AA$ molecular sieves and the solution was cooled to -10 ${ }^{\circ} \mathrm{C}$. TMSOTf $(0.10 \mathrm{~mL}, 0.56 \mathrm{mmol})$ was added, followed by the dropwise addition of a solution of donor trichloroacetimidate $3(2.7 \mathrm{~g}, 3.94 \mathrm{mmol})$ in $\mathrm{Et}_{2} \mathrm{O}(10 \mathrm{~mL})$. Stirring was continued for $20 \mathrm{~min}$ and then triethylamine $(5 \mathrm{~mL})$ was added. The mixture was filtered through Celite, the filtrate concentrated and the resulting residue was purified by chromatography (hexane-EtOAc; 4:1). The pure product (5) was obtained as a colourless oil $(2.26 \mathrm{~g})$ in $76 \%$ yield. $[\alpha]_{\mathrm{D}}+78.0(c$ 1.0 $\mathrm{CHCl}_{3}$ ). ${ }^{1} \mathrm{H}$ NMR (500 MHz): 8.07-8.01 (4H, m, ArH), 7.63-7.60 (1H, m, ArH), 7.52-7.46 (4H, m, ArH), 6.95-6.93 (2H, m, ArH), 5.63 (1H, d, J $2.5 \mathrm{~Hz}, \mathrm{H}-4), 5.56$ (1H, t, J $10.0 \mathrm{~Hz}, \mathrm{H}-2)$, 5.18 (1H, d, J 3.0 Hz, H-1'), 4.78-4.74 (2H, m, H-1, $\left.\mathrm{PhCH}_{2}\right), 4.63-4.61\left(3 \mathrm{H}, \mathrm{PhCH}_{2}\right), 4.45-4.40$ $\left(2 \mathrm{H}, \mathrm{m}, \mathrm{H}-6, \mathrm{PhCH}_{2}\right), 4.40-4.28$ (4H, m, H-6, $\left.\mathrm{PhCH}_{2}\right), 4.10$ (1H, dd, J 3.0, 10.0 Hz, H-3), 3.933.85 (3H, m, H-2', H-5, H-5'), 3.55 (1H, dd, J 3.0, 10.0 Hz, H-3'), 3.40 (1H, dd, J 7.0, 9.5 Hz, H6'), 3.24-3.22 (1H, m, H-4'), 3.17 (1H, dd, J 5.3, 9.5 Hz, H-6'), 2.28 (3H, s, $\left.\mathrm{PhCH}_{3}\right), 1.88(3 \mathrm{H}, \mathrm{s}$, $\left.\mathrm{OC}(\mathrm{O}) \mathrm{CH}_{3}\right) .{ }^{13} \mathrm{C} \mathrm{NMR}(125 \mathrm{MHz}): 170.4,166.0,164.9,129.3-138.1,94.0,87.5,78.8,76.5$, 75.6, 74.9, 73.3, 73.2(5), 73.1(9), 72.8, 70.0, 69.5, 69.1, 65.2, 62.8, 21.1, 20.5. HRMS-ESI (m/z) calcd for $\mathrm{C}_{62} \mathrm{H}_{63} \mathrm{O}_{13} \mathrm{SNa}$ : 1081.3803. Found $[\mathrm{M}+\mathrm{Na}]^{+} 1081.3798$.

7-Octen-1-yl 2,3,4,6-tetra- $O$-benzyl- $\alpha$-D-galactopyranosyl-(1 $\rightarrow$ 3)-4- $O$-acetyl-2,6-di- $O$ benzoyl- $\beta$-D-galactopyranosyl-( $\rightarrow 4)$-2,3,6-tri- $O$-benzyl- $\beta$-D-glucopyranoside

(10).

Thioglycoside $5(1.2 \mathrm{~g}, 1.13 \mathrm{mmol})$ was dissolved in acetone $(30 \mathrm{~mL})$ and cooled to $0{ }^{\circ} \mathrm{C} . \mathrm{N}$ Bromosuccinimide $(0.4 \mathrm{~g}, 2.27 \mathrm{mmol})$ was added and the mixture stirred in air until the reaction was complete. The reaction mixture was then diluted with EtOAc $(150 \mathrm{~mL})$ and extracted with 
saturated aqueous $\mathrm{NaHCO}_{3}(2 \times 100 \mathrm{~mL})$, dried $\left(\mathrm{MgSO}_{4}\right)$ and evaporated. The oil obtained was purified via column chromatography (hexane-EtOAc 2:1) and the resulting hemiacetal 6 (0.5 g, $0.52 \mathrm{mmol})$ was then dissolved in dry $\mathrm{CH}_{2} \mathrm{Cl}_{2}(20 \mathrm{~mL})$ and cooled to $0{ }^{\circ} \mathrm{C}$. Trichloroacetonitrile $(0.21 \mathrm{~mL}, 2.1 \mathrm{mmol})$ and DBU $(31 \mu \mathrm{L}, 0.21 \mathrm{mmol})$ were added and the solution stirred at $0{ }^{\circ} \mathrm{C}$ for $4 \mathrm{~h}$. After warming to room temperature, the solvent was removed under reduced pressure and the residue obtained was purified via flash chromatography (hexane-EtOAc 6:1) to give imidate 7 as a colourless oil (0.50 g, 73\% yield over 2 steps), which was used immediately. Acceptor $8(0.21 \mathrm{~g}, 0.36 \mathrm{mmol})$ was dissolved in $\mathrm{CH}_{2} \mathrm{Cl}_{2}(\mathrm{~mL})$ containing $4 \AA$ molecular sieves and the solution was cooled to $-10{ }^{\circ} \mathrm{C}$. TMSOTf $(55 \mu \mathrm{L}, 0.03 \mathrm{mmol})$ was added, followed by the dropwise addition of a solution of donor trichloroacetimidate $7(0.50 \mathrm{~g}, 0.19 \mathrm{mmol})$ in $\mathrm{CH}_{2} \mathrm{Cl}_{2}$ $(10 \mathrm{~mL})$. Stirring was continued for $20 \mathrm{~min}$ and then triethylamine $(5 \mathrm{~mL})$ was added. The mixture was filtered through Celite, the filtrate concentrated and the resulting residue was purified by chromatography (hexane-EtOAc, 4:1). The pure product (10) was obtained as a colourless oil (0.48 g) in 88\% yield. ${ }^{1} \mathrm{H}$ NMR (500 MHz): 8.07-8.01 (4H, m, ArH), 7.63-7.60 $(1 \mathrm{H}, \mathrm{m}, \mathrm{ArH}), 7.52-7.46(4 \mathrm{H}, \mathrm{m}, \mathrm{ArH}), 6.95-6.93(2 \mathrm{H}, \mathrm{m}, \mathrm{ArH}), 5.85-5.77\left(1 \mathrm{H}, \mathrm{m}, \mathrm{CH}=\mathrm{CH}_{2}\right)$, 5.57 (1H, d, J 3.2 Hz, H-4'), 5.54 (1H, dd, J 8.1, 10.2 Hz, H-2'), 5.19 (1H, d, J 3.4 Hz, H-1' '), $5.03\left(1 \mathrm{H}, \mathrm{d}, J 11.1 \mathrm{~Hz}, \mathrm{PhCH}_{2}\right), 5.02-4.94\left(2 \mathrm{H}, \mathrm{m}, \mathrm{CH}=\mathrm{CH}_{2}\right), 4.93-4.88\left(3 \mathrm{H}, \mathrm{m}, \mathrm{H}-1^{\prime}, \mathrm{PhCH}_{2}\right)$, $4.76\left(1 \mathrm{H}, \mathrm{d}, J 11.5 \mathrm{~Hz}, \mathrm{PhCH}_{2}\right), 4.74\left(1 \mathrm{H}, \mathrm{d}, J 11.1 \mathrm{~Hz}, \mathrm{PhCH}_{2}\right), 4.71-4.65$ (3H, m, H-1, $\left.\mathrm{PhCH}_{2}\right), 4.50-4.48$ (2H, PhCH $\left.{ }_{2}\right), 4.44$ (1H, d, J 11.8 Hz, PhCH $\left.\mathrm{H}_{2}\right), 4.32-4.30$ (4H, m, H-1, H-6', $\left.\mathrm{PhCH}_{2}\right), 4.27-4.24$ (1H, m, H-6'), $4.17\left(2 \mathrm{H}, \mathrm{d}, J 6.8 \mathrm{~Hz}, \mathrm{PhCH}_{2}\right), 4.01(1 \mathrm{H}, \mathrm{dd}, J 3.3,10.2 \mathrm{~Hz}$, H-3'), 3.97-3.92 (2H, m, H-2'', H-5'), $3.88\left(1 \mathrm{H}, \mathrm{td}, J 6.5,9.5 \mathrm{~Hz}, \mathrm{OCH}_{2}\left(\mathrm{CH}_{2}\right)_{5} \mathrm{CH}=\mathrm{CH}_{2}\right), 3.84$ (1H, t, J 6.5 Hz, H-6 ), 3.66-3.58 (5H, m, H-6, H-5'', H-4, H-3, H-3' '), 3.47 (1H, dt, J 6.8, 9.5 $\left.\mathrm{Hz}, \mathrm{OCH}_{2}\left(\mathrm{CH}_{2}\right)_{5} \mathrm{CH}=\mathrm{CH}_{2}\right), 3.43-3.40\left(2 \mathrm{H}, \mathrm{m}, \mathrm{H}-2, \mathrm{H}-6^{\prime \prime}\right), 3.32(1 \mathrm{H}, \mathrm{ddd}, J 1.9,4.3,9.8 \mathrm{~Hz}, \mathrm{H}-$ 5), 3.20-3.17 (2H, m, H-4"', H-6"'), 2.06-2.02 (2H, m, $\left.\mathrm{OCH}_{2}\left(\mathrm{CH}_{2}\right)_{5} \mathrm{CH}=\mathrm{CH}_{2}\right), 1.86(3 \mathrm{H}, \mathrm{s}$, $\left.\mathrm{OC}(\mathrm{O}) \mathrm{CH}_{3}\right), \quad 1.65-1.61 \quad\left(2 \mathrm{H}, \quad \mathrm{m}, \quad \mathrm{OCH}_{2}\left(\mathrm{CH}_{2}\right)_{5} \mathrm{CH}=\mathrm{CH}_{2}\right), \quad 1.41-1.26 \quad(6 \mathrm{H}, \quad \mathrm{m}$, $\left.\mathrm{OCH}_{2}\left(\mathrm{CH}_{2}\right)_{5} \mathrm{CH}=\mathrm{CH}_{2}\right) .{ }^{13} \mathrm{C}$ NMR $(125 \mathrm{MHz}): 169.9,165.4,164.4,126.3-138.8,113.9,103.3$, 103.2, 100.3, 93.5, 83.7, 82.4, 81.4, 81.3(8), 78.3, 75.2, 74.9, 74.6, 74.5(7), 74.4, 74.3, 74.1, 74.0, 73.3, 73.1, 72.9, 72.8, 71.7, 71.3, 71.2(7), 70.6, 70.0, 69.8, 69.5, 69.4, 68.9, 53.1, 29.4, 29.3, 28.6, 28.5, 28.4(6), 25.7, 25.6, 20.1. HRMS-ESI $(\mathrm{m} / \mathrm{z})$ calcd for $\mathrm{C}_{91} \mathrm{H}_{98} \mathrm{O}_{19} \mathrm{Na}$ : 1517.6595. Found $[\mathrm{M}+\mathrm{Na}]^{+} 1517.6600$.

7-Octen-1-yl 2,3,4,6-tetra- $O$-benzyl- $\alpha$-D-galactopyranosyl-(1 $\rightarrow 3)$-4- $O$-acetyl-2,6-di- $O$ benzoyl- $\beta$-D-galactopyranosyl-( $1 \rightarrow 4)$-2-azido-2-deoxy-3,6-di- $O$-benzyl- $\beta$-D-glucopyranoside (11). The reaction was carried out as described for the preparation of $\mathbf{1 0}$ using acceptor 9 and trichloroacetimidate 7 to provide 11 in $85 \%$ yield. ${ }^{1} \mathrm{H}$ NMR $(500 \mathrm{MHz})$ : 8.07-8.01 (4H, m, ArH), 7.63-7.60 (1H, m, ArH), 7.52-7.46 (4H, m, ArH), 6.95-6.93 (2H, m, ArH), 5.85-5.77 $\left(1 \mathrm{H}, \mathrm{m}, \mathrm{OCH}_{2}\left(\mathrm{CH}_{2}\right)_{5} \mathrm{CH}=\mathrm{CH}_{2}\right), 5.54\left(1 \mathrm{H}, \mathrm{d}, J 3.4 \mathrm{~Hz}, \mathrm{H}-4^{\prime}\right), 5.50\left(1 \mathrm{H}, \mathrm{dd}, J 8.0,10.0 \mathrm{~Hz}, \mathrm{H}-2^{\prime}\right)$, $5.14\left(1 \mathrm{H}, \mathrm{d}, J 3.4 \mathrm{~Hz}, \mathrm{H}-1^{\prime \prime}\right), 5.02\left(1 \mathrm{H}, \mathrm{d}, J 11.1 \mathrm{~Hz}, \mathrm{PhCH}_{2}\right), 4.99-4.90$ (2H, m, $\left.\mathrm{OCH}_{2}\left(\mathrm{CH}_{2}\right)_{5} \mathrm{CH}=\mathrm{CH}_{2}\right), 4.82-4.72\left(3 \mathrm{H}, \mathrm{m}, \mathrm{H}-1^{\prime}, \mathrm{PhCH}_{2}\right), 4.66-4.60$ (3H, m, H-1, PhCH $), 4.49$ $\left(1 \mathrm{H}, \mathrm{d}, J 11.8 \mathrm{~Hz}, \mathrm{PhCH}_{2}\right), 4.44-4.40\left(2 \mathrm{H}, \mathrm{m}, \mathrm{PhCH}_{2}\right), 4.31-4.26$ (4H, m, $\left.\mathrm{PhCH}_{2}\right), 4.15-4.01$ $\left(3 \mathrm{H}, \mathrm{m}, \mathrm{PhCH}_{2}\right), 3.98-3.89$ (3H, m, H-2'", H-5, H-6), 3.82-3.78 (2H, m, OCH$\left.\left(\mathrm{CH}_{2}\right)_{5} \mathrm{CH}=\mathrm{CH}_{2}\right)$, 
3.62-3.52 (5H, m), $3.42\left(1 \mathrm{H}, \mathrm{dt}, J 6.8,9.5 \mathrm{~Hz}, \mathrm{OCH}_{2}\left(\mathrm{CH}_{2}\right)_{5} \mathrm{CH}=\mathrm{CH}_{2}\right), 3.39-3.30$ (4H, m), 3.21$3.17(3 \mathrm{H}, \mathrm{m}), 2.06-2.02\left(2 \mathrm{H}, \mathrm{m}, \mathrm{OCH}_{2}\left(\mathrm{CH}_{2}\right)_{5} \mathrm{CH}=\mathrm{CH}_{2}\right), 1.86\left(3 \mathrm{H}, \mathrm{s}, \mathrm{OC}(\mathrm{O}) \mathrm{CH}_{3}\right), 1.65-1.62$ $\left(2 \mathrm{H}, \mathrm{m}, \mathrm{OCH}_{2}\left(\mathrm{CH}_{2}\right)_{5} \mathrm{CH}=\mathrm{CH}_{2}\right), 1.41-1.26\left(6 \mathrm{H}, \mathrm{m}, \mathrm{OCH}_{2}\left(\mathrm{CH}_{2}\right)_{5} \mathrm{CH}=\mathrm{CH}_{2}\right) .{ }^{13} \mathrm{C}$ NMR $(125$ $\mathrm{MHz})$ : 170.2, 165.9, 165.5, 127.3-139.1, 114.2, 102.0, 100.5, 94.1, 81.0, 78.8, 76.5, 76.4, 75.6, 75.0, 74.9, 74.7, 74.5, 73.5, 73.5, 73.4, 73.2(5), 73.1(6), 72.2, 71.6, 71.0, 70.2, 69.9, 69.3, 67.9, $65.9,64.8(6), 64.5,61.5, \quad 33.7,29.5,28.8,25.8, \quad 20.5$. HRMS-ESI $(\mathrm{m} / z)$ calcd for $\mathrm{C}_{84} \mathrm{H}_{91} \mathrm{~N}_{3} \mathrm{O}_{18} \mathrm{Na}: 1452.619$. Found $[\mathrm{M}+\mathrm{Na}]^{+} 1452.6167$.

7-Octen-1-yl 2,3,4,6-tetra- $O$-benzyl- $\alpha$-D-galactopyranosyl-(1 $\rightarrow 3)$-4- $O$-acetyl-2,6-di- $O$ benzoyl- $\beta$-D-galactopyranosyl- $(1 \rightarrow 4)-2-N$-acetamido-2-deoxy-3,6-di- $O$-benzyl- $\beta$-D-

glucopyranoside (12). Compound $10(0.44 \mathrm{~g}, 0.31 \mathrm{mmol})$ was dissolved in pyridine $(4 \mathrm{~mL})$, thioacetic acid $(2 \mathrm{~mL})$ was added and the mixture was stirred at room temperature for $24 \mathrm{~h}$. The solvent was then removed in vacuo and the residue purified via column chromatography (hexane- EtOAc 1:1) to give 12 as a colourless oil $\left(0.37 \mathrm{~g}, 83 \%\right.$ yield). ${ }^{1} \mathrm{H} \mathrm{NMR}$ (500 MHz, $\left.\mathrm{CHCl}_{3}\right):$ 8.07-8.00 (4H, m, ArH), 7.63-7.60 (1H, m, ArH), 7.53-7.49 (1H, m, ArH), 7.47-7.44 $(2 \mathrm{H}, \mathrm{m}, \mathrm{ArH}), 7.37-7.21(33 \mathrm{H}, \mathrm{m}, \mathrm{ArH}), 7.16-7.14(2 \mathrm{H}, \mathrm{m}, \mathrm{ArH}), 6.01(1 \mathrm{H}, \mathrm{d}, J 8.5 \mathrm{~Hz},-$ $\mathrm{NHAc}), 5.85-5.77\left(1 \mathrm{H}, \mathrm{m}, \mathrm{OCH}_{2}\left(\mathrm{CH}_{2}\right)_{5} \mathrm{CH}=\mathrm{CH}_{2}\right), 5.60(1 \mathrm{H}, \mathrm{d}, J 3.5 \mathrm{~Hz}), 5.50(1 \mathrm{H}, \mathrm{dd}, J$ 8.0, $10.5 \mathrm{~Hz}), 5.20(1 \mathrm{H}, \mathrm{d}, J 3.5 \mathrm{~Hz}), 5.03-4.93(2 \mathrm{H}, \mathrm{m}), 4.79-4.62\left(7 \mathrm{H}, \mathrm{m}, \mathrm{PhCH}_{2}\right.$ x 6, H-1), 4.53$4.44\left(4 \mathrm{H}, \mathrm{m}, \mathrm{PhCH}_{2}\right), 4.36-4.31(4 \mathrm{H}, \mathrm{m}), 4.24(1 \mathrm{H}, \mathrm{dd}, J 6.7,11.2 \mathrm{~Hz}), 4.09$ (1H, dd, J 3.5, 10.5 $\mathrm{Hz}), 4.06(1 \mathrm{H}, \mathrm{t}, J 5.5 \mathrm{~Hz}), 3.97-3.95(2 \mathrm{H}, \mathrm{m}), 3.90(1 \mathrm{H}, \mathrm{t}, J 5.7 \mathrm{~Hz}), 3.84(1 \mathrm{H}, \mathrm{m}), 3.46-3.71$ $(6 \mathrm{H}, \mathrm{m}), 3.47(1 \mathrm{H}, \mathrm{dd}, J 6.5,9.5 \mathrm{~Hz}), 3.36(1 \mathrm{H}, \mathrm{br} \mathrm{s}), 3.31(1 \mathrm{H}, \mathrm{dd}, J 6.0 \mathrm{~Hz}, 9.5 \mathrm{~Hz}), 3.00(1 \mathrm{H}$, $\mathrm{m}), \quad 2.07-2.01 \quad\left(5 \mathrm{H}, \mathrm{m}, \mathrm{OC}(\mathrm{O}) \mathrm{CH}_{3}\right), 1.88\left(3 \mathrm{H}, \mathrm{s}, \mathrm{OC}(\mathrm{O}) \mathrm{CH}_{3}\right), \quad 1.46-1.38 \quad(2 \mathrm{H}, \mathrm{m}$, $\left.\mathrm{OCH}_{2}\left(\mathrm{CH}_{2}\right)_{5} \mathrm{CH}=\mathrm{CH}_{2}\right), 1.36-1.17\left(6 \mathrm{H}, \mathrm{m}, \mathrm{OCH}_{2}\left(\mathrm{CH}_{2}\right)_{5} \mathrm{CH}=\mathrm{CH}_{2}\right) .{ }^{13} \mathrm{C} \mathrm{NMR}: 170.2,170.1$, $165.9,165.5,138.60,138.57,138.5,138.11,138.08,133.4,133.3,127.4-129.8,114.2,100.5$, 99.6, 94.0, 78.8, 77.3, 76.4, 76.2, 74.6, 73.6, 73.4, 73.1, 72.7, 71.6, 71.3, 71.2, 69.9, 69.4, 69.3, $64.3,61.7,33.7,29.4,28.9,25.8,23.4,20.5$. HRMS-ESI $(m / z)$ calcd for $\mathrm{C}_{85} \mathrm{H}_{95} \mathrm{NO}_{19} \mathrm{Na}$ : 1468.639. Found $[\mathrm{M}+\mathrm{Na}]^{+} 1468.6386$.

7-Octen-1-yl $\alpha$-D-galactopyranosyl-(1 $\rightarrow 3)-\beta$-D-galactopyranosyl-( $\rightarrow 4)-\beta$-D-glucopyranoside (13). Compound $10(100 \mathrm{mg}, 66.9 \mu \mathrm{mol})$ was dissolved in THF $(2 \mathrm{~mL})$ containing $\mathrm{CH}_{3} \mathrm{OH}$ $(100 \mu \mathrm{L})$. The resulting solution was added to liquid ammonia $(\sim 5 \mathrm{~mL})$ containing $\mathrm{Na}$ metal at $78{ }^{\circ} \mathrm{C}$. After $1 \mathrm{~h}, \mathrm{CH}_{3} \mathrm{OH}(5 \mathrm{~mL})$ was added to the reaction mixture, the ammonia was blown off using a stream of air and the solution evaporated to dryness. The residue was taken up in $\mathrm{CH}_{3} \mathrm{OH}$ $(50 \mathrm{~mL})$, filtered and evaporated. The residue was subjected to $\mathrm{C} 18$ chromatography, using gradient elution $\left(100 \% \mathrm{H}_{2} \mathrm{O} \rightarrow 50: 50 \mathrm{H}_{2} \mathrm{O}-\mathrm{CH}_{3} \mathrm{OH}\right)$ to give $\mathbf{1 3}$ as a white solid (40 mg, 97\% yield). ${ }^{1} \mathrm{H}$ NMR (500 MHz, CD $\left.{ }_{3} \mathrm{OD}\right): 5.85-5.77\left(1 \mathrm{H}, \mathrm{m}, \mathrm{OCH}_{2}\left(\mathrm{CH}_{2}\right)_{5} \mathrm{CH}=\mathrm{CH}_{2}\right), 5.02(1 \mathrm{H}, \mathrm{d}, J$ $\left.1.8 \mathrm{~Hz}, \mathrm{H}-1^{\prime \prime}\right), 5.00-4.83\left(2 \mathrm{H}, \mathrm{m}, \mathrm{OCH}_{2}\left(\mathrm{CH}_{2}\right)_{5} \mathrm{CH}=\mathrm{CH}_{2}\right), 4.41\left(1 \mathrm{H}, \mathrm{d}, J 7.3 \mathrm{~Hz}, \mathrm{H}-1^{\prime}\right), 4.27(1 \mathrm{H}$, d, J 7.8 Hz, H-1), 4.21 (1H, app t, J $\left.6.5 \mathrm{~Hz} \mathrm{H}-5^{\prime \prime}\right), 4.03(1 \mathrm{H}, \mathrm{d}, J 1.8 \mathrm{~Hz}), 3.91-3.50$ (15H, m), $3.38(1 \mathrm{H}, \mathrm{m}), 3.23(1 \mathrm{H}, \mathrm{dd}, J 8.1,8.6 \mathrm{~Hz}, \mathrm{H}-2), 2.07-2.02\left(2 \mathrm{H}, \mathrm{m}, \mathrm{OCH}_{2}\left(\mathrm{CH}_{2}\right)_{5} \mathrm{CH}=\mathrm{CH}_{2}\right), 1.62-$ $1.59\left(2 \mathrm{H}, \mathrm{m}, \mathrm{OCH}_{2}\left(\mathrm{CH}_{2}\right)_{5} \mathrm{CH}=\mathrm{CH}_{2}\right), 1.43-1.29\left(6 \mathrm{H}, \mathrm{m}, \mathrm{OCH}_{2}\left(\mathrm{CH}_{2}\right)_{5} \mathrm{CH}=\mathrm{CH}_{2}\right) .{ }^{13} \mathrm{C}$ NMR: 142.6, 117.3, 107.7, 105.3, 100.3, 83.9, 82.6, 79.1, 76.8, 74.8, 73.9, 73.7, 73.6, 73.1, 72.7, 69.2, 
65.3, 65.0, 59.3, 37.4, 33.2, 32.7, 32.5, 29.5, 25.5. HRMS-ESI $(\mathrm{m} / \mathrm{z})$ calcd for $\mathrm{C}_{26} \mathrm{H}_{46} \mathrm{O}_{16} \mathrm{Na}$ : 637.2678. Found $[\mathrm{M}+\mathrm{Na}]^{+} 637.2677$.

7-Octen-1-yl $\quad \alpha$-D-galactopyranosyl-( $1 \rightarrow 3)$ - $\beta$-D-galactopyranosyl-( $1 \rightarrow 4)-\beta$-D-2-deoxy-2acetamidoglucopyranoside (14). The deprotection carried out as described for the preparation of 13, starting from 12 to yield 14 in $85 \%$ yield. ${ }^{1} \mathrm{H}$ NMR (500 MHz, $\left.\mathrm{CD}_{3} \mathrm{OD}\right): 5.85-5.77(1 \mathrm{H}$, $\left.\mathrm{m}, \quad \mathrm{OCH}_{2}\left(\mathrm{CH}_{2}\right)_{5} \mathrm{CH}=\mathrm{CH}_{2}\right), \quad 5.02\left(1 \mathrm{H}, \mathrm{d}, \quad J \quad 1.8 \mathrm{~Hz}, \mathrm{H}-1^{\prime \prime}\right), \quad 5.00-4.83$ (2H, m, $\left.\mathrm{OCH}_{2}\left(\mathrm{CH}_{2}\right)_{5} \mathrm{CH}=\mathrm{CH}_{2}\right), 4.45\left(1 \mathrm{H}, \mathrm{d}, J 7.1 \mathrm{~Hz}, \mathrm{H}-1^{\prime}\right), 4.40(1 \mathrm{H}, \mathrm{d}, J 8.2 \mathrm{~Hz}, \mathrm{H}-1), 4.21(1 \mathrm{H}$, app t, $J$ 6.1 Hz H-5'), 4.02 (1H, d, J 2.5 Hz), 3.93-3.57 (15H, m), 3.45 (1H, m), 3.37 (1H, m), 3.31 $(1 \mathrm{H}, \mathrm{dd}, J 6.0 \mathrm{~Hz}, 9.5 \mathrm{~Hz}), 3.00(1 \mathrm{H}, \mathrm{m}), 2.06-2.02\left(2 \mathrm{H}, \mathrm{m}, \mathrm{OCH}_{2}\left(\mathrm{CH}_{2}\right)_{5} \mathrm{CH}=\mathrm{CH}_{2}\right), 1.95(3 \mathrm{H}, \mathrm{s}$, $\left.\mathrm{OC}(\mathrm{O}) \mathrm{CH}_{3}\right), \quad 1.55-1.50 \quad\left(2 \mathrm{H}, \quad \mathrm{m}, \quad \mathrm{OCH}_{2}\left(\mathrm{CH}_{2}\right)_{5} \mathrm{CH}=\mathrm{CH}_{2}\right), \quad 1.41-1.28 \quad(6 \mathrm{H}, \quad \mathrm{m}$, $\left.\mathrm{OCH}_{2}\left(\mathrm{CH}_{2}\right)_{5} \mathrm{CH}=\mathrm{CH}_{2}\right) .{ }^{13} \mathrm{C}$ NMR: 142.6, 117.3, 107.7, 105.3, 100.3, 83.9, 82.6, 79.1, 76.8, 74.8, 73.9, 73.7, 73.6, 73.1, 72.7, 69.2, 65.3, 65.0, 59.3, 37.4, 33.2, 32.7, 32.5, 29.5, 25.5. HRMS-ESI $(\mathrm{m} / \mathrm{z})$ calcd for $\mathrm{C}_{28} \mathrm{H}_{49} \mathrm{NO}_{16} \mathrm{Na}$ : 678.2944. Found $[\mathrm{M}+\mathrm{Na}]^{+}$678.2932.

8-[(2-Aminoethyl)thio]-1-octyl $\alpha$-D-galactopyranosyl-( $\rightarrow 3)$ - $\beta$-D-galactopyranosyl-( $1 \rightarrow 4)-\beta$ D-glucopyranosyl hydrochloride (15). Alkene $13(12 \mathrm{mg}, 0.020 \mathrm{mmol})$ and cysteamine hydrochloride $(17 \mathrm{mg}, 0.15 \mathrm{mmol})$ were placed in a quartz tube and dry $\mathrm{CH}_{3} \mathrm{OH}(0.5 \mathrm{~mL})$ added. The solution was degassed and the tube irradiated with UV light for $2 \mathrm{~h}$. The solution was then evaporated and subjected to $\mathrm{C}_{18}$ column chromatography, gradient elution $(100 \% 0.5 \%$ aqueous $\mathrm{AcOH} \rightarrow 50: 50 \mathrm{H}_{2} \mathrm{O}-0.5 \%$ aqueous $\left.\mathrm{AcOH}\right)$ to give 15 as a white solid (13 mg, 90\% yield). ${ }^{1} \mathrm{H}$ NMR (500 MHz, CD 3 OD): 5.02 (1H, d, J $\left.1.8 \mathrm{~Hz}, \mathrm{H}-1^{\prime \prime}\right), 4.41$ (1H, d, J 7.1 Hz, H-1'), 4.28 (1H, d, J 7.8 Hz, H-1), 4.21 (1H, app t, J $\left.6.1 \mathrm{~Hz}, \mathrm{H}-5^{\prime \prime}\right), 4.03(1 \mathrm{H}, \mathrm{d}, J 1.6 \mathrm{~Hz}), 3.93-3.47$ (16H, m), 3.42-3.37 (1H, m), $3.23(1 \mathrm{H}, \mathrm{t}, J 8.3 \mathrm{~Hz}, \mathrm{H}-2), 3.09\left(2 \mathrm{H}, \mathrm{t}, J 6.8 \mathrm{~Hz}, \mathrm{~S}\left(\mathrm{CH}_{2}\right)_{2} \mathrm{NH}_{3} \mathrm{Cl}\right), 2.77(2 \mathrm{H}$, $\left.\mathrm{t}, \quad .6 .8 \mathrm{~Hz}, \mathrm{OCH}_{2}\left(\mathrm{CH}_{2}\right)_{5} \mathrm{CH}_{2} \mathrm{~S}\left(\mathrm{CH}_{2}\right)_{2} \mathrm{NH}_{3} \mathrm{Cl}\right), 2.57\left(2 \mathrm{H}, \mathrm{t}, J 7.3 \mathrm{~Hz}, \mathrm{CH}_{2} \mathrm{~S}\left(\mathrm{CH}_{2}\right)_{2} \mathrm{NH}_{3} \mathrm{Cl}\right), 1.61-$ $1.51\left(4 \mathrm{H}, \mathrm{m}, \mathrm{OCH}_{2}\left(\mathrm{CH}_{2}\right)_{5} \mathrm{CH}_{2} \mathrm{~S}\right), 1.42-1.32\left(6 \mathrm{H}, \mathrm{m}, \mathrm{OCH}_{2}\left(\mathrm{CH}_{2}\right)_{5} \mathrm{CH}_{2} \mathrm{~S}\right) .{ }^{13} \mathrm{C} \mathrm{NMR}: 105.1$, 104.1, 88.7, 81.0, 80.0, 76.7, 76.5, 74.8, 72.3, 71.4, 71.2, 70.2, 66.7, 62.8, 62.4, 40.0, 36.6, 33.1, $32.7,32.5,31.3,30.6,30.7,30.3,30.2,29.7,27.0,23.0$. HRMS-ESI $(\mathrm{m} / z)$ calcd for $\mathrm{C}_{28} \mathrm{H}_{54} \mathrm{NO}_{16} \mathrm{~S}: 692.3158$. Found $[\mathrm{M}+\mathrm{H}]^{+} 692.3149$.

8-[(2-Aminoethyl)thio]-1-octyl $\alpha$-D-galactopyranosyl-( $1 \rightarrow 3)-\beta$-D-galactopyranosyl-(1 $\rightarrow 4)-\beta$ D-2-deoxy-2-acetamido glucopyranosyl hydrochloride (16). The photochemical addition was carried out as described for the preparation of 15 starting from 14, providing 16 in $97 \%$ yield. ${ }^{1} \mathrm{H}$ NMR (500 MHz, CD 3 OD): 5.03 (1H, br s, H-1' '), 4.42 (1H, d, J 6.9 Hz, H-1'), 4.38 (1H, d, J 6.3 Hz, H-1), 4.21 (1H, t, J 6.0 Hz H-5' ), 4.03 (1H, br s), 3.93-3.47 (17H, m), 3.42-3.37 (1H, m,), $3.09\left(2 \mathrm{H}, \mathrm{t}, J 6.8 \mathrm{~Hz}, \mathrm{~S}\left(\mathrm{CH}_{2}\right)_{2} \mathrm{NH}_{3} \mathrm{Cl}\right), 2.77\left(2 \mathrm{H}, \mathrm{t}, J 6.8 \mathrm{~Hz}, \mathrm{~S}\left(\mathrm{CH}_{2}\right)_{2} \mathrm{NH}_{3} \mathrm{Cl}\right), 2.57(2 \mathrm{H}, \mathrm{t}, J 7.3$ $\left.\mathrm{Hz}, \mathrm{OCH}_{2}\left(\mathrm{CH}_{2}\right)_{5} \mathrm{CH}_{2} \mathrm{~S}\right), 1.96\left(3 \mathrm{H}, \mathrm{s}, \mathrm{C}(\mathrm{O}) \mathrm{CH}_{3}\right), 1.61-1.51\left(4 \mathrm{H}, \mathrm{m}, \mathrm{OCH}_{2}\left(\mathrm{CH}_{2}\right)_{5} \mathrm{CH}_{2} \mathrm{~S}\right), 1.42-$ $1.32\left(6 \mathrm{H}, \mathrm{m}, \mathrm{OCH}_{2}\left(\mathrm{CH}_{2}\right)_{5} \mathrm{CH}_{2} \mathrm{~S}\right) .{ }^{13} \mathrm{C} \mathrm{NMR}: 105.1,102.8,97.8,81.4,80.0,76.7,76.6,74.3,72.3$, 71.4, 71.7, 71.1, 70.7, 70.1, 66.7, 62.8, 62.5, 62.4(8), 62.1, 56.7, 40.0, 32.5, 30.6, 30.5, 30.3, 30.2, 29.7, 27.0, 23.0. HRMS-ESI $(\mathrm{m} / \mathrm{z})$ calcd for $\mathrm{C}_{30} \mathrm{H}_{57} \mathrm{~N}_{2} \mathrm{O}_{16} \mathrm{~S}: 733.3423$. Found $[\mathrm{M}+\mathrm{H}]^{+}$ 733.3422 .

6-[[2-[[8-( $\alpha$-D-Galactopyranosyl- $(1 \rightarrow 3)-\beta$-D-galactopyranosyl-( $1 \rightarrow 4)-\beta$-D-glucopyranosyloxy)octyl]thio]ethyl]amino]-6-oxo-hexanoic acid $p$-nitrophenyl ester (1). Compound 15 (6 
mg, $8.2 \mu \mathrm{mol})$ was dissolved in $\mathrm{CH}_{3} \mathrm{OH}(10 \mathrm{~mL})$ and treated with Amberlite IRA $400\left(\mathrm{HO}^{-}\right.$ form) to convert the hydrochloride salt to the amine. The solution was then filtered, concentrated and dried under vacuum. The residue was then dissolved in dimethylacetamide $(1 \mathrm{~mL})$ and treated with di-p-nitrophenyl adipate $(12 \mathrm{mg}, \mathrm{mmol})$. The solution was stirred for $4 \mathrm{~h}$, then evaporated at $35{ }^{\circ} \mathrm{C}$ under high vacuum. The resulting residue was purified using $\mathrm{C}_{18}$ column chromatography, gradient elution $\left(100 \% 0.5 \%\right.$ aqueous $\mathrm{AcOH} \rightarrow 50: 50 \mathrm{H}_{2} \mathrm{O}-0.5 \%$ aqueous $\mathrm{AcOH}$ ) to give 1 ( $7 \mathrm{mg}, 90 \%$ yield), which was lyophilized in $0.5 \%$ aqueous acetic acid to give a white solid. ${ }^{1} \mathrm{H}$ NMR (500 MHz, $\left.\mathrm{CD}_{3} \mathrm{OD}\right): 8.28(2 \mathrm{H}, \mathrm{d}, J 9.1 \mathrm{~Hz}), 7.36(2 \mathrm{H}, \mathrm{d}, J 9.1 \mathrm{~Hz}), 5.02$ (1H, br s, H-1), $4.78(1 \mathrm{H}, J 3.4 \mathrm{~Hz}), 4.48(1 \mathrm{H}, \mathrm{d}, J 7.5 \mathrm{~Hz}), 4.20(1 \mathrm{H}, \mathrm{t}, J 6.0 \mathrm{~Hz}), 3.90-3.51$ $(15 \mathrm{H}, \mathrm{m}), 3.50-3.31(4 \mathrm{H}, \mathrm{m}), 2.66\left(2 \mathrm{H}, \mathrm{t}, J .7 .0 \mathrm{~Hz}, \mathrm{~S}\left(\mathrm{CH}_{2}\right)_{2} \mathrm{~N}\right), 2.61\left(2 \mathrm{H}, \mathrm{t}, J 6.9 \mathrm{~Hz}, \mathrm{~S}\left(\mathrm{CH}_{2}\right)_{2} \mathrm{~N}\right)$, $2.52\left(2 \mathrm{H}, \mathrm{t}, J 7.3 \mathrm{~Hz}, \mathrm{PNPOC}(\mathrm{O}) \mathrm{CH}_{2}\right), 2.24\left(2 \mathrm{H}, \mathrm{t}, J 6.9 \mathrm{~Hz}, \mathrm{NC}(\mathrm{O}) \mathrm{CH}_{2}\right), 1.89-1.79(4 \mathrm{H}, \mathrm{m}$, PNPOC $\left.(\mathrm{O}) \mathrm{CH}_{2}\left(\mathrm{CH}_{2}\right)_{2}\right)$, 1.59-1.53 (4H, m, $\left.\mathrm{OCH}_{2}\left(\mathrm{CH}_{2}\right)_{5} \mathrm{CH}_{2} \mathrm{~S}\right), \quad 1.41-1.29 \quad(6 \mathrm{H}, \quad \mathrm{m}$, $\left.\mathrm{OCH}_{2}\left(\mathrm{CH}_{2}\right)_{5} \mathrm{CH}_{2} \mathrm{~S}\right)$. HRMS-ESI $(\mathrm{m} / \mathrm{z})$ calcd for $\mathrm{C}_{40} \mathrm{H}_{64} \mathrm{~N}_{2} \mathrm{O}_{21} \mathrm{SNa}$ : 963.3614. Found $[\mathrm{M}+\mathrm{Na}]^{+}$ 963.3617.

6-[[2-[[8-( $\alpha$-D-Galactopyranosyl-( $1 \rightarrow 3)$ - $\beta$-D-galactopyranosyl-( $1 \rightarrow 4)-\beta$-D-2-deoxy-2-acetamidoglucopyranosyloxy)octyl]thio]ethyl]amino]-6-oxo-hexanoic acid $p$-nitrophenyl ester (2). Prepared as described for the preparation of $\mathbf{1}$ starting from 16, giving 2 in $90 \%$ yield as a white solid. ${ }^{1} \mathrm{H}$ NMR (500 MHz, $\left.\mathrm{CD}_{3} \mathrm{OD}\right): 8.28(2 \mathrm{H}, \mathrm{d}, J 9.1 \mathrm{~Hz}), 7.36(2 \mathrm{H}, \mathrm{d}, J 9.1 \mathrm{~Hz}), 5.02$ $(1 \mathrm{H}$, br s, H-1), $4.42(1 \mathrm{H}, \mathrm{d}, J 7.3 \mathrm{~Hz}), 4.39(1 \mathrm{H}, \mathrm{d}, J 8.1 \mathrm{~Hz}), 4.20(1 \mathrm{H}, \mathrm{t}, J 6.0 \mathrm{~Hz}), 3.90-3.51$ $(15 \mathrm{H}, \mathrm{m}), 3.52-3.31(4 \mathrm{H}, \mathrm{m}), 2.66\left(2 \mathrm{H}, \mathrm{t}, J 7.0 \mathrm{~Hz}, \mathrm{~S}\left(\mathrm{CH}_{2}\right)_{2} \mathrm{~N}\right), 2.61\left(2 \mathrm{H}, \mathrm{t}, J 6.9 \mathrm{~Hz}, \mathrm{~S}\left(\mathrm{CH}_{2}\right)_{2} \mathrm{~N}\right)$, $2.52\left(2 \mathrm{H}, \mathrm{t}, J 7.3 \mathrm{~Hz}, \operatorname{PNPOC}(\mathrm{O}) \mathrm{CH}_{2}\right), 2.24\left(2 \mathrm{H}, \mathrm{t}, J 6.9 \mathrm{~Hz}, \mathrm{NC}(\mathrm{O}) \mathrm{CH}_{2}\right), 1.96(3 \mathrm{H}, \mathrm{s}$, $\left.\mathrm{C}(\mathrm{O}) \mathrm{CH}_{3}\right), 1.89-1.79\left(4 \mathrm{H}, \mathrm{m}, \operatorname{PNPOC}(\mathrm{O}) \mathrm{CH}_{2}\left(\mathrm{CH}_{2}\right)_{2}\right)$ 1.59-1.53 (4H, m, $\left.\mathrm{OCH}_{2}\left(\mathrm{CH}_{2}\right)_{5} \mathrm{CH}_{2} \mathrm{~S}\right)$, 1.41-1.29 (6H, m, $\left.\mathrm{OCH}_{2}\left(\mathrm{CH}_{2}\right)_{5} \mathrm{CH}_{2} \mathrm{~S}\right)$. HRMS-ESI $(\mathrm{m} / \mathrm{z})$ calcd for $\mathrm{C}_{40} \mathrm{H}_{67} \mathrm{~N}_{3} \mathrm{O}_{21} \mathrm{SNa}$ : 1004.3880 . Found $[\mathrm{M}+\mathrm{Na}]^{+} 1004.3877$.

\section{Acknowledgements}

Funding for this work was obtained via a CIHR team grant (RMF92091), an NSERC/CIHR CHRP grant (CHRPJ350946-08) and the Alberta Glycomics Centre

\section{References}

1. Galili, U. Immunol. Cell. Biol. 2005, 83, 674.

2. Galili, U. Immunol. Today 1993, 14, 480.

3. Lai, L.; Kolber-Simonds, D.; Park, K. W.; Cheong, H. T.; Greenstein, J. L.; Im, G. S.; Samuel, M.; Bonk, A.; Rieke, A.; Day, B. N.; Murphy, C. N.; Carter, D. B.; Hawley, R. J.; Prather, R. S. Science 2002, 295, 1089. 
4. Phelps, C. J.; Koike, C.; Vaught, T. D.; Boone, J.; Wells, K. D.; Chen, S. H.; Ball, S.; Specht, S. M.; Polejaeva, I. A.; Monahan, J. A.; Jobst, P. M.; Sharma, S. B.; Lamborn, A. E.; Garst, A. S.; Moore, M.; Demetris, A. J.; Rudert, W. A.; Bottino, R.; Bertera, S.; Trucco, M.; Starzl, T. E.; Dai, Y.; Ayares, D. L. Science 2003, 299, 411.

5. Tazelaar, H. D.; Byrne, G. W.; McGregor, C. G. Transplantation 2011, 91, 968.

6. Agostino, M.; Sandrin, M. S.; Thompson, P. E.; Ramsland, P. A.; Yuriev, E. Biopolymers 2011, 96, 193.

7. Brenner, P.; Keller, M.; Beiras-Fernandez, A.; Uchita, S.; Kur, F.; Thein, E.; Wimmer, C.; Hammer, C.; Schmoeckel, M.; Reichart, B. Ann. Transplant. 2010, 15, 30.

8. Mangold, A.; Lebherz, D.; Papay, P.; Liepert, J.; Hlavin, G.; Lichtenberger, C.; Adami, A.; Zimmermann, M.; Klaus, D.; Reinisch, W.; Ankersmit, H. J. Transplant Proc. 2011, 43, 3964.

9. Commins, S. P.; James, H. R.; Kelly, L. A.; Pochan, S. L.; Workman, L. J.; Perzanowski, M. S.; Kocan, K. M.; Fahy, J. V.; Nganga, L. W.; Ronmark, E.; Cooper, P. J.; Platts-Mills, T. A. J. Allergy. Clin. Immunol. 2011, 127, 1286.

10. Fontan, M. P.; Manez, R.; Rodriguez-Carmona, A.; Peteiro, J.; Martinez, V.; GarciaFalcon, T.; Domenech, N. Am. J. Kidney Dis. 2006, 48, 972.

11. Larkin, J. M.; Norsworthy, P. J.; A'Hern, R. P.; Eisen, T. G.; Gore, M. E.; Porter, C. D. Melanoma Res. 2006, 16, 157.

12. Galili, U. Clin. Dev. Immunol. 2011, 134020.

13. Deguchi, T.; Tanemura, M.; Miyoshi, E.; Nagano, H.; Machida, T.; Ohmura, Y.; Kobayashi, S.; Marubashi, S.; Eguchi, H.; Takeda, Y.; Ito, T.; Mori, M.; Doki, Y.; Sawa, Y. Cancer Res. 2010, 70, 5259.

14. Galili, U. A., M. R.; Sondel, P. M.; Wigglesworth, K.; Sullivan, M.; Whalen, Giles F. Cancers 2010, 2, 773.

15. Rossi, G. R.; Mautino, M. R.; Awwad, D. Z.; Husske, K.; Lejukole, H.; Koenigsfeld, M.; Ramsey, W. J.; Vahanian, N.; Link, C. J. J. Immunother. 2008, 31, 545.

16. Abdel-Motal, U. M.; Wang, S.; Awad, A.; Lu, S.; Wigglesworth, K.; Galili, U. Vaccine 2010, 28, 1758.

17. Galili, U. Transplantation 2004, 78, 1093.

18. Macher, B. A.; Galili, U. Biochem. Biophys. Acta 2008, 1780, 75.

19. Galili, U.; Ishida, H.; Tanabe, K.; Toma, H. Transplantation 2002, 74, 1574.

20. Slaney, A. M.; Wright, V. A.; Meloncelli, P. J.; Harris, K. D.; West, L. J.; Lowary, T. L.; Buriak, J. M. ACS Appl. Mater. Interfaces. 2011, 3, 1601.

21. Fang, J. W.; Li, J.; Chen, X.; Zhang, Y. N.; Wang, J. Q.; Guo, Z. M.; Zhang, W.; Yu, L. B.; Brew, K.; Wang, P. G. J. Am. Chem. Soc. 1998, 120, 6635.

22. Qian, X. P.; Sujino, K.; Otter, A.; Palcic, M. M.; Hindsgaul, O. J. Am. Chem. Soc. 1999, $121,12063$.

23. Weignerova, L.; Sedmera, P.; Hunkova, Z.; Halada, P.; Kren, V.; Casali, M.; Riva, S. Tetrahedron Lett. 1999, 40, 9297. 
24. Wang, Y. H.; Yan, Q. Y.; Wu, J. P.; Zhang, L. H.; Ye, X. S. Tetrahedron 2005, 61, 4313.

25. Wang, Y. H.; Huang, X. F.; Zhang, L. H.; Ye, X. S. Org. Lett. 2004, 6, 4415.

26. Litjens, R. E. J. N.; Hoogerhout, P.; Filippov, D. V.; Codee, J. D. C.; van den Bos, L. J.; van den Berg, R. J. B. H. N.; Overkleeft, H. S.; van der Marel, G. A. J. Carbohydr. Chem. 2005, 24, 755 .

27. Gege, C.; Kinzy, W.; Schmidt, R. R. Carbohydr. Res. 2000, 328, 459.

28. Zhang, W.; Wang, J. Q.; Li, J.; Yu, L. B.; Wang, P. G. J. Carbohydr. Chem. 1999, 18, 1009.

29. Schmidt, R. R.; Michel, J. Angew. Chim. 1980, 92, 763.

30. Demchenko, A. V.; Rousson, E.; Boons, G.-J. Tetrahedron Lett. 1999, 40, 6523.

31. Meloncelli, P. J.; Lowary, T. L. Carbohydr. Res. 2010, 345, 2305.

32. Shangguan, N.; Katukojvala, S.; Greenberg, R.; Williams, L. J. J. Am. Chem. Soc. 2003, $125,7754$.

33. Dondoni, A.; Massi, A.; Nanni, P.; Roda, A. Chem. Eur. J. 2009, 15, 11444.

34. Wu, X. Y.; Ling, C. C.; Bundle, D. R. Org. Lett. 2004, 6, 4407. 